\title{
Giant neutron halos in the non-relativistic mean field approach
}

\author{
M. Grasso, ${ }^{1,2,3}$ S. Yoshida, ${ }^{1,4}$ N. Sandulescu,,${ }^{5,6}$ and N. Van Giai ${ }^{1}$ \\ ${ }^{1}$ Institut de Physique Nucléaire, 15 rue Georges Clémenceau, F-91406 Orsay Cedex, France \\ ${ }^{2}$ Dipartimento di Fisica e Astronomia, Via Santa Sofia 64, I-95123 Catania, Italy \\ ${ }^{3}$ INFN, Sezione di Catania, Via Santa Sofia 64, I-95123 Catania, Italy \\ ${ }_{4}^{4}$ Science Research Center, Hosei University, 2-17-1 Fujimi, Chiyoda, Tokyo 102-8160, Japan \\ ${ }^{5}$ Service de Physique Nucléaire, CEA-DAM Ile-de-France, BP 12, F-91680 Bruyères-le-Châtel, France \\ ${ }^{6}$ Institute for Physics and Nuclear Engineering, P.O. Box MG-6, 76900 Bucharest, Romania
}

\begin{abstract}
Giant neutron halos in medium-heavy nuclei are studied in the framework of the Hartree-FockBogoliubov (HFB) approach with Skyrme interactions. The appearance of such structures depends sensitively on the effective interaction adopted. This is illustrated by comparing the predictions of SLy4 and SkI4 in the Ca and Zr isotopic chains. The former force gives no halo effect, the latter predicts a neutron halo in the $\mathrm{Zr}$ chain with $\mathrm{A}>122$ due to the weakly bound orbitals $3 p 1 / 2$ and $3 p 3 / 2$. The structure of the halo is analyzed in terms of the occupation probabilities of these orbitals and their partial contributions to the neutron density. The anti-halo effect in Ni and Zr isotopes is also discussed by comparing the occupation probabilities of Hartree-Fock neutron single-particle states near the Fermi energy with the corresponding HFB quasiparticle states.
\end{abstract}

PACS numbers: 21.10.Gv, 21.10.Pc, 21.60.Jz, 27.40.+z, 27.50.+e, 27.60.+j

\section{INTRODUCTION}

In the present years several projects for the construction of a new generation of radioactive beam facilities are in progress (see, e.g.,Ref. [1]). Such facilities will permit to investigate the properties of unstable nuclei situated close to the drip line regions. From the theoretical side, many efforts are devoted to perform accurate predictions to locate the proton and neutron drip lines as well as to describe the behavior of unstable nuclei. Unfortunately, theoretical predictions in exotic regions of the nuclear chart can be rather model dependent.

Self-consistent mean field methods are well suited theoretical tools for describing medium and heavy nuclei. There are two main lines of investigations based on the mean field approach, namely the relativistic mean field (RMF) method where effective Lagrangians are treated in the tree approximation, and the nonrelativistic Hartree-Fock (HF) method using effective interactions like the Gogny and Skyrme forces. Recent reviews can be found in Refs. 2, 3]. When approaching the drip lines, one deals with open shell nuclei where the effects of pairing correlations become quite important, especially for such properties as the tails of matter distributions. The pairing correlations can be described by the non-relativistic Hartree-Fock-Bogoliubov (HFB) theory [4, 5], or by the relativistic Hartree-Bogoliubov (RHB) theory [6], and one must add to the models a phenomenological pairing interaction acting in the particleparticle channel.

Furthermore, the chemical potential $\lambda$ becomes close to zero in the vicinity of drip lines and it is necessary to treat properly the contributions of the quasiparticle continuum when evaluating the pairing correlations [5]. Thus, the most appropriate approach for such cases is to solve the self-consistent mean field equations in coordinate space, and this is the method we use to obtain the results of this work.

Important discrepancies are often found in the position of the neutron drip line predicted by different models. For instance, these differences clearly appear in the neutron drip line of $\mathrm{Ni}$ isotopes calculated in relativistic [7] and non-relativistic [5] approaches. Even among different parametrizations of effective Skyrme interactions used in HFB approach one finds different drip line predictions, as illustrated in the recent work of Ref. [8] and in the results of the present work. While waiting for new experimental data which will help to discriminate among the available models it can be interesting to analyze the reasons for some of these discrepancies. This is one of the aims of this paper.

A very interesting phenomenon has been recently predicted within the RHB approach, the formation of a neutron giant halo (with up to six neutrons involved) in some very neutron-rich isotopes. By plotting the neutron distribution radius with respect to $A$ a kink appears where the halo structure starts being formed. This effect has been found to be particularly strong in Ca (with $A>60$ ) 9] and $\mathrm{Zr}$ isotopes (with $A>122$ ) [10]. These predictions are based on the NLSH parametrization, and similar results are obtained with the TM1 parametrization 11. The giant halo phenomenon is also found in the near-drip line Zr isotopes if one uses the NL3 parametrization in an RMF plus resonant continuum BCS 12. Until recently, the giant halo effect in mediumheavy nuclei has been much less investigated within the non-relativistic mean field approach, apart from Ref. [8] where the study of halo is concentrated on the Ca isotopes. In this paper, we focus our discussion on the $\mathrm{Zr}$ isotopes where we find that, in the HFB approach with Skyrme forces the position of the neutron drip line depends on the particular force adopted and consequently, the giant halo effect seems to be more model dependent than in relativistic approaches. 
A well known phenomenon for which relativistic and non-relativistic predictions are quite different is the charge isotope shift in $\mathrm{Pb}$ isotopes: the kink in the charge radius at the shell closure $N=126$ is not reproduced by non-relativistic calculations with the Gogny interaction 13. or with standard Skyrme parametrizations while it can be well reproduced by RMF calculations. Reinhard and Flocard 14] have analyzed this discrepancy with a special attention to the spin-orbit parametrization of Skyrme interactions. New parametrizations have been proposed in order to reproduce the observed kink of the charge isotope shift. If one introduces two parameters in the spin-orbit part of the energy functional instead of one as in the standard Skyrme interactions, it is possible to obtain a spin-orbit potential with isovector properties similar to that of RMF. Then, a fitting procedure of all the Skyrme force parameters is performed to produce new parametrizations which can describe well a chosen set of nuclei and also reproduce the kink in the $\mathrm{Pb}$ isotope shift. In the present calculations we have selected the parametrization SkI4, and for comparison purposes we also use the more standard set SLy4.

In this work we investigate the giant halo effect in $\mathrm{Ca}$ and $\mathrm{Zr}$ isotopes within the non-relativistic SkyrmeHartree-Fock-Bogoliubov mean field approach. We find that the neutron drip line of $\mathrm{Zr}$ isotopes calculated with SkI4 is far enough to give rise to a giant halo phenomenon. On the other hand, neither SLy4 nor SkI4 can produce bound $\mathrm{Ca}$ isotopes which are enough neutronrich to lead to a halo effect. The anti-halo effect due to pairing correlations [15] is discussed for $\mathrm{Zr}$ and $\mathrm{Ni}$ isotopes by comparing HFB results with the corresponding HF ones.

The article is organized as follows. In Sec. II we briefly describe the theoretical framework. In Sec. III we present the calculated two-neutron separation energies, neutron radii and single-particle spectra for neutron-rich $\mathrm{Ca}$ and $\mathrm{Zr}$ isotopes and we compare our non-relativistic results with the corresponding RHB results obtained with the NLSH parametrization [9, 10]. In Sec. III we consider in more detail the case of $\mathrm{Zr}$ isotopes and investigate giant halo and anti-halo effects by analyzing occupation probabilities, neutron density profiles and radii. The anti-halo effect is also studied for $\mathrm{Ni}$ isotopes. In Sec. IV our conclusions are drawn.

\section{THEORETICAL FRAMEWORK}

The theoretical framework used in this paper is the Hartree-Fock-Bogoliubov (HFB) approach. For local two-body forces and spherical symmetry the HFB equations have the following form:

$$
\begin{aligned}
& {[h(\mathbf{r})-\lambda] u(E, \mathbf{r})+\Delta(\mathbf{r}) v(E, \mathbf{r})=E u(E, \mathbf{r})} \\
& \Delta(\mathbf{r}) u(E, \mathbf{r})-[h(\mathbf{r})-\lambda] v(E, \mathbf{r})=E v(E, \mathbf{r}),
\end{aligned}
$$

where $\lambda$ is the Fermi energy, $h$ is the sum of the kinetic energy and the HF mean field potential, and $\Delta$ is the pairing potential; $u$ and $v$ are the upper and lower components of the quasiparticle wave function associated with the energy $E$.

The quasiparticle spectrum contains a discrete part for $E$ less than $-\lambda$ and a continuous part for $E$ above $-\lambda$. To calculate the continuum spectrum the HFB equations should be solved with scattering type boundary conditions for the upper components of the HFB wave functions [5]. Since the continuum-HFB calculations are rather heavy, usually the continuum spectrum is discretized by imposing box boundary conditions, i.e., the condition that the HFB wave functions vanish at a given distance from the nucleus. This is sufficient for our present purpose provided that the box radius is properly chosen. We have checked that our results obtained with a box radius of $20 \mathrm{fm}$ are very close to those of a full continuum calculation.

In the HFB calculations presented in this paper the mean field is calculated with a Skyrme type force while for the pairing channel we use a zero range interaction with the following density dependence:

$$
V\left(\mathbf{r}_{1}-\mathbf{r}_{2}\right)=V_{0}\left[1-x\left(\frac{\rho(r)}{\rho_{0}}\right)^{\gamma}\right] \delta\left(\mathbf{r}_{1}-\mathbf{r}_{2}\right)
$$

We have adopted the values $\rho_{0}=0.16 \mathrm{fm}^{-3}, x=0.5, \gamma=1$. The strength $V_{0}$ is chosen so as to reproduce the gaps extracted from the odd-even mass differences ( in the regions where such experimental data are available).

As for the Skyrme interaction, we use two forces, i.e., SLy4 and SkI4. The force SLy4, which includes constraints coming from the neutron matter equation of state, is commonly employed for the description of neutron-rich nuclei. The force SkI4 has a spin-orbit potential similar to the RMF models and it includes in the fitting protocol the requirement to reproduce the charge isotope shift for $\mathrm{Pb}$ isotopes. Due to these characteristics one expects that this Skyrme force will predict for the nuclei close to the drip line properties similar to those of relativistic models. We are particularly interested in the giant halo which might be formed in heavy nuclei close to the neutron drip line. Neutron giant halos were predicted by the RHB calculations in $\mathrm{Ca}$ and $\mathrm{Zr}$ regions for $A>60$ and $A>122$, respectively [7]. Thus, the pairing strengths $V_{0}$ are adjusted to be $-365 \mathrm{MeV} \cdot \mathrm{fm}^{3}$ and -290 $\mathrm{MeV} \cdot \mathrm{fm}^{3}\left(-350 \mathrm{MeV} \cdot \mathrm{fm}^{3}\right.$ and $\left.-300 \mathrm{MeV} \cdot \mathrm{fm}^{3}\right)$ for the $\mathrm{Ca}$ and $\mathrm{Zr}$ isotopes calculated with SLy4 (SkI4).

The Skyrme model predictions will be analyzed in the next section by using the HFB approach. The HFB calculations are performed in spherical symmetry, like in the RHB approach. 


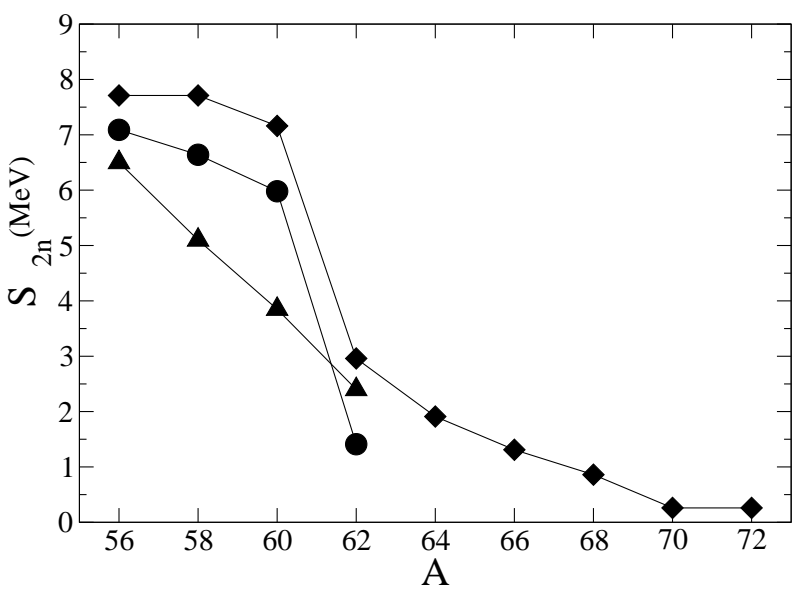

FIG. 1: Two-neutron separation energies for Ca isotopes calculated with SLy4-HFB (triangles), SkI4-HFB (circles) and RHB (diamonds). The RHB results correspond to Ref. [9].

\section{RESULTS OF HFB CALCULATIONS}

\section{A. Separation energies and neutron radii}

The two-neutron separation energy is defined as

$$
S_{2 n}(N, Z)=E(N, Z)-E(N-2, Z)
$$

where $E(N, Z)$ is the total energy of the isotope with $N$ neutrons and $Z$ protons. The two-neutron separation energies for $\mathrm{Ca}$ and $\mathrm{Zr}$ isotopes are shown in Figs. 1 and 2. In these figures are displayed only the separation energies for the bound nuclei (i.e., those having a negative chemical potential in the HFB calculations). The results correspond to the box-HFB calculations, which give practically the same $S_{2 n}$ values as continuum-HFB calculations.

The most important fact we can observe in Fig. 1 is the large difference between the drip line location predicted by the Skyrme-HFB with SLy4 or SkI4, and the RHB calculations of Ref. [9] for the Ca isotopes. It can be seen that, for both Skyrme forces the drip line is located at ${ }^{62} \mathrm{Ca}$, while for $\mathrm{RHB}$ calculations the drip line extends up to ${ }^{72} \mathrm{Ca}$. However, it is found in Ref. 8] that the two-neutron separation energy is still positive in ${ }^{78} \mathrm{Ca}$ with the interaction $\mathrm{SkM}^{*}$. This strong sensitivity of the drip line location to the particular Skyrme force makes difficult the study of neutron-rich $\mathrm{Ca}$ isotopes. In the $\mathrm{Zr}$ isotopes there are also differences among the model predictions. As seen in Fig.2, for the force SLy4 the drip line is located at ${ }^{122} \mathrm{Zr}$ while for SkI4 at ${ }^{138} \mathrm{Zr}$. The latter result is similar to the RHB one where the drip line is located at ${ }^{140} \mathrm{Zr}$.

Next we analyze the neutron radii, shown in Fig. 3. As expected, for $\mathrm{Ca}$ isotopes the HFB calculations with the interactions SLy4 and SKI4 do not predict a giant halo because the neutron drip line is reached before the halo

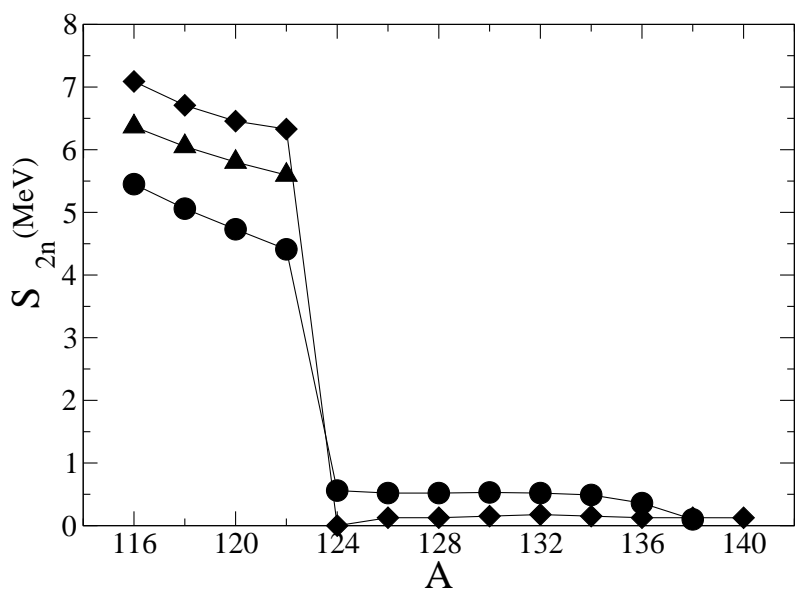

FIG. 2: Two-neutron separation energies for $\mathrm{Zr}$ isotopes. The symbols are the same as in Fig. 1. The RHFB results correspond to Ref.[10].

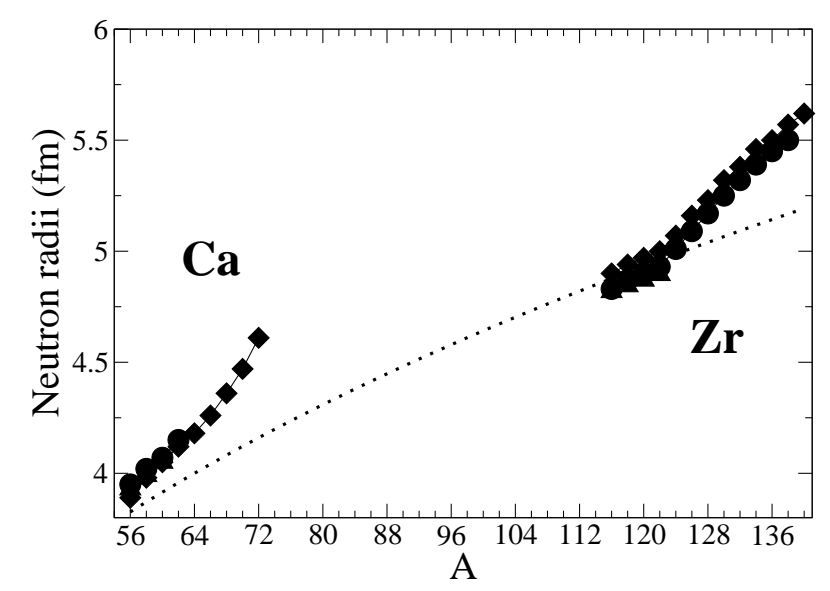

FIG. 3: Neutron radii for $\mathrm{Ca}$ and $\mathrm{Zr}$ isotopes. The symbols are the same as in Fig. 1 and the RHB results correspond to Ref. [9, 10]. Radii calculated with the formula $r_{0} A^{1 / 3}\left(r_{0}=1\right.$ fm) are shown by a dotted line.

structure starts to be formed. The same is happening for $\mathrm{Zr}$ isotopes if one uses the SLy4 force. However, for the force SkI4 the situation is very different. Thus, as seen in Fig. 3, the neutron radii given by the SkI4-HFB calculations show a change of slope around $N=82$ and then follow the RHB results.

To understand better the behaviour of the radii, in Figs. 4 and 5 are shown the HF energies of the bound states close to the Fermi level. For Ca isotopes we can see that, apart from a different splitting of the $1 f$ states, the two Skyrme forces give a rather similar structure for the bound spectrum. In both cases the state $1 g 9 / 2$ is not bound, at variance with the RMF calculations in which this state becomes weakly bound at $A=62$. Due to this fact the drip line in the RHB calculations is extended up to the region where the giant halo can be formed, in 


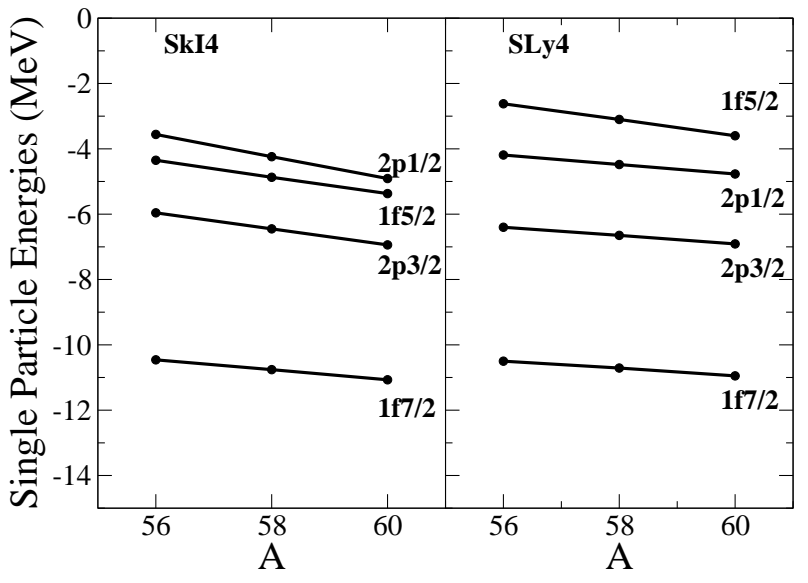

FIG. 4: SkI4-HF (left) and SLy4-HF (right) neutron single particle energies for bound states in $\mathrm{Ca}$ isotopes.

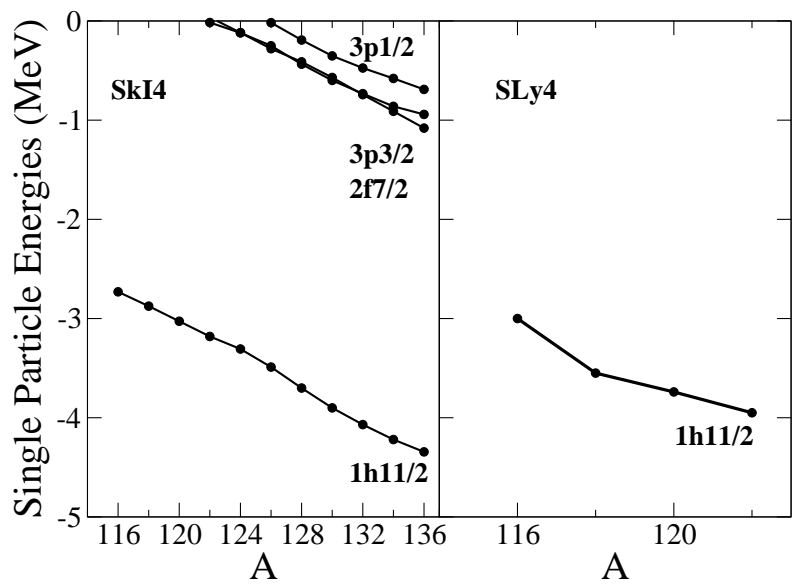

FIG. 5: SkI4-HF (left) and SLy4-HF (right) neutron single particle energies for bound states in $\mathrm{Zr}$ isotopes.

contrast with the HFB calculations of Ca isotopes based on SLy4 or SkI4. It must be noted, however, that this tendency is not obeyed by the $\mathrm{SkM}^{*}$ parametrization which gives a bound 1 $199 / 2$ orbital in the Ca isotopes with $\mathrm{A} \geq 60$ resulting in a drip line located at higher $\mathrm{A}[\underline{8}]$.

For $\mathrm{Zr}$ isotopes the structure of the bound spectrum is not the same for the two Skyrme forces. We can observe that, for the force SLy4 there is only one bound state close to the Fermi level, i.e., $1 h 11 / 2$, while for the force SkI4 the states $2 f 7 / 2,3 p 3 / 2$ and $3 p 1 / 2$ appear as bound states as well. These three weakly bound states have a similar structure in the RMF calculations. This explains why the SkI4-HFB and the RHB calculations provide a similar halo in $\mathrm{Zr}$ isotopes. How these weakly bound HF states contribute to the halo structure in the presence of pairing correlations is discussed in the next subsection.

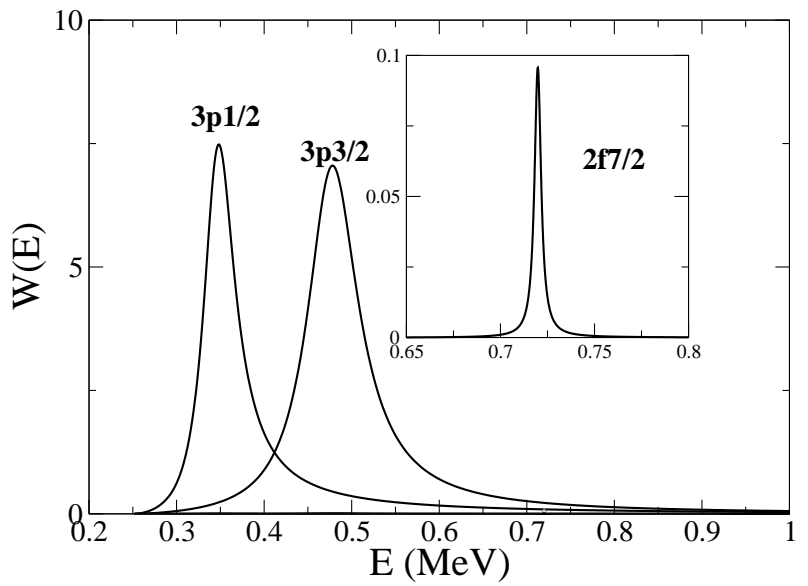

FIG. 6: Occupation profiles $W(E)$ (in units of $\mathrm{MeV}^{-1}$ ) for the states $3 p 1 / 2,3 p 3 / 2$ and $2 f 7 / 2$ in ${ }^{132} \mathrm{Zr}$, calculated with SkI4-HFB.

\section{B. The structure of the giant halo}

In the presence of pairing correlations the bound single-particle states shown in Fig. 5 are becoming quasiparticle resonances. To describe them properly we have solved the HFB equations with scattering type boundary conditions. In order to reduce the numerical effort we have employed scattering boundary conditions only for the quasiparticle states with the energy $-\lambda<E<15$ $\mathrm{MeV}$ while for the other states we have used box boundary conditions.

With the continuum HFB solutions we can calculate how the occupation probability is changing in the region around a resonance. This information is provided by the quantity

$$
W(E)=\int_{0}^{R} d r r^{2} v_{E}^{2}(r)
$$

where $v$ is the lower component of the HFB wave function and $R$ is take equal to $20 \mathrm{fm}$. As an example, in Fig. 6 we show the values of $\mathrm{W}(\mathrm{E})$ corresponding to the states $3 p 1 / 2,3 p 3 / 2$ and $2 f 7 / 2$ in the nucleus ${ }^{132} \mathrm{Zr}$. In this nucleus the quasiparticle continuum starts at the energy $E=-\lambda=0.251 \mathrm{MeV}$.

Integrating the function $\mathrm{W}(\mathrm{E})$ over an energy interval in which it has a significant value we can associate to each resonance an occupation probability $n$, i.e.,

$$
n=\int_{E_{1}}^{E_{2}} W(E) d E
$$

The occupation probabilities of the relevant resonant states in $\mathrm{Zr}$ isotopes are shown in Fig. 7. It can be seen that the occupation probabilities corresponding to the weakly bound states are increasing progressively when passing from ${ }^{124} \mathrm{Zr}$ to ${ }^{138} \mathrm{Zr}$. Thus these states contribute significantly to the pairing correlations. 


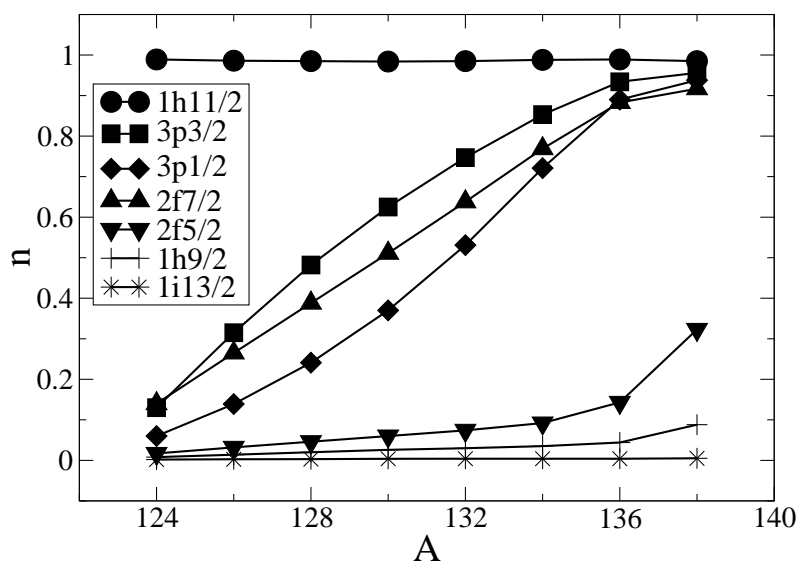

FIG. 7: Occupation probabilities for $\mathrm{Zr}$ isotopes, calculated with SkI4-HFB.

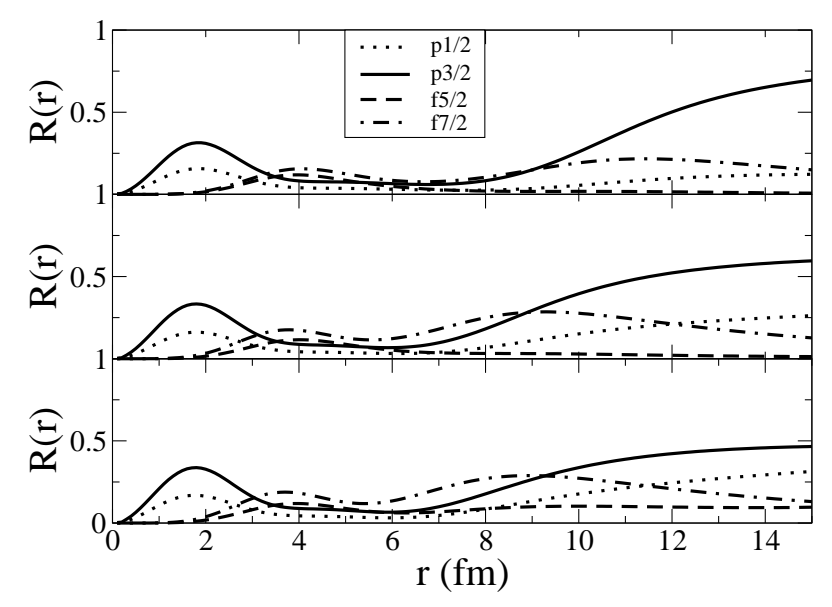

FIG. 8: Contributions of different $(l, j)$ channels to the total neutron density for ${ }^{124} \mathrm{Zr}$ (top), ${ }^{132} \mathrm{Zr}$ (middle) and ${ }^{138} \mathrm{Zr}$ (bottom). The interaction is SkI4.

In order to analyze the structure of the halo, in Fig. 8 we plot the quantity

$$
R_{l j}(r)=\frac{\rho_{l j}(r)}{\rho(r)}
$$

where $\rho$ and $\rho_{l j}$ are the total density and the density corresponding to the channel $(l, j)$, respectively. From Fig. 8 we can clearly see that at large distances the dominant contribution to the neutron density is given by the $p$ states, which are less confined by the centrifugal barrier compared to the other states with higher $(l, j)$ values. This structure of the giant halo obtained by using the SkI4-HFB model is very similar to that given by the relativistic calculations [7, 12].

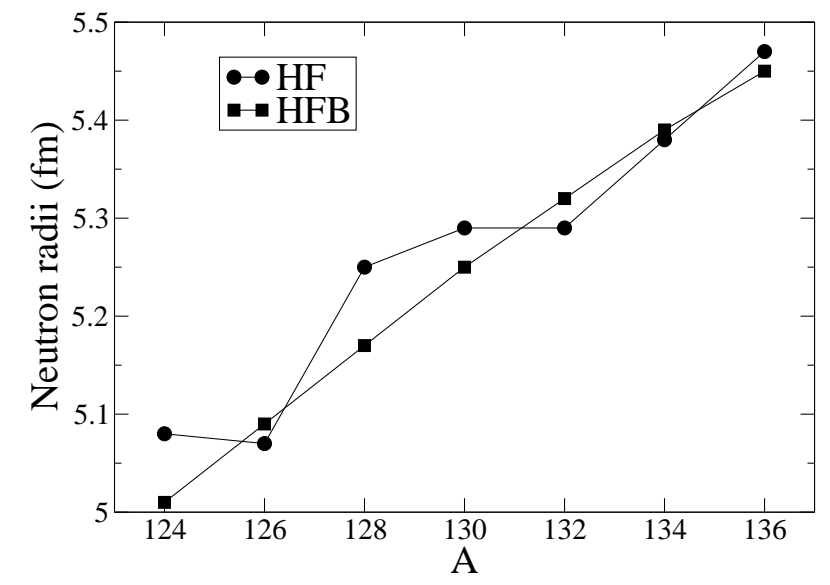

FIG. 9: HF (circles) and HFB (squares) neutron radii for $\mathrm{Zr}$ isotopes. The interaction is SkI4.

\section{The anti-halo effect}

We turn now to the analysis of the so-called "anti-halo effect", which was mainly discussed in light nuclei close to the neutron drip line [15]. This effect is associated with a reduction of the neutron radii by the pairing correlations. Here, we will show that in fact the pairing correlations not only decrease but can also increase the neutron radii in nuclei close to the drip line.

A strong effect of the pairing correlations upon the neutron radii is expected when close to the Fermi level one finds weakly bound $s$ or $p$ states together with states with higher angular momenta (weakly bound or resonances). As an illustration we present below the case of $\mathrm{Zr}$ and $\mathrm{Ni}$ isotopes.

In $\mathrm{Zr}$ isotopes the pairing correlations affect the neutron halo through the weakly bound $p$ and $f$ states. In Fig. 9 are shown the neutron radii obtained in the HF and HFB calculations performed with the force SkI4. We observe that in some cases $(A=124,128,130,136)$ the $\mathrm{HF}$ radii are larger than the $\mathrm{HFB}$ ones while in other cases $(A=126,132,134)$ the contrary happens. This behaviour can be easily understood if one considers the occupancy of the states $3 p 1 / 2,3 p 3 / 2$ and $2 f 7 / 2$ in $\mathrm{HF}$ and HFB calculations shown in Table I and Fig.7. We observe that for $A=124$ the state $3 p 3 / 2$ is half occupied while $2 f 7 / 2$ is empty in HF. When pairing correlations are switched on, the occupancy of the state $3 p 3 / 2$ is reduced and that of the $2 f 7 / 2$ is enhanced. Since the state $2 f 7 / 2$ has a smaller radial extension than the state $3 p 3 / 2$ due to the centrifugal barrier, the HFB radius is reduced compared to the HF radius. Similar considerations apply for $A=128$ and 130 . For $A=136$ the three states are completely occupied in $\mathrm{HF}$. The HFB radius is thus smaller than the $\mathrm{HF}$ one due to the reduction of the occupancy of the two $p$ states when pairing is switched on.

On the other hand, for $A=126$ the situation is opposite: in $\mathrm{HF}$ approximation the state $3 p 3 / 2$ is empty and 


\begin{tabular}{|c|c|c|c|c|c|c|c|}
\hline$A$ & 124 & 126 & 128 & 130 & 132 & 134 & 136 \\
\hline $3 p 1 / 2$ & 0 & 0 & 0 & 0 & 0 & 0 & 2 \\
\hline $3 p 3 / 2$ & 2 & 0 & 4 & 4 & 2 & 4 & 4 \\
\hline $2 f 7 / 2$ & 0 & 4 & 2 & 4 & 8 & 8 & 8 \\
\hline
\end{tabular}

TABLE I: Number of neutrons in the states $3 p 1 / 2,3 p 3 / 2$ and $2 f 7 / 2$ in $\mathrm{HF}$ as a function of $N$ in $\mathrm{Zr}$ isotopes.

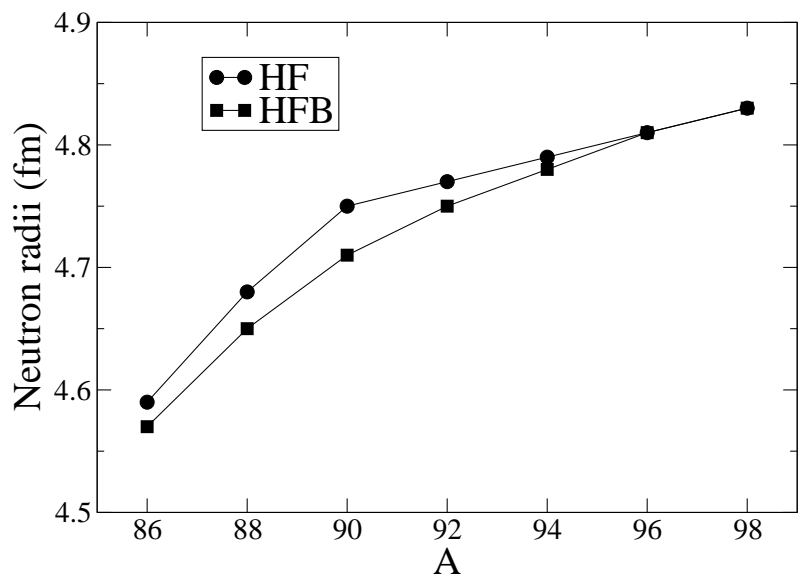

FIG. 10: HF (circles) and HFB (squares) neutron radii for $\mathrm{Ni}$ isotopes. The interaction is SkI4.

the state $2 f 7 / 2$ is partially occupied. Thus, in this case pairing enhances the occupation of the state $3 p 3 / 2$ and reduces that of the state $2 f 7 / 2$. Consequently, the radius becomes larger in HFB than in HF. A similar mechanism applies for $A=132$ where the state $2 f 7 / 2$ is completely occupied in $\mathrm{HF}$ and $3 p 3 / 2$ only partially occupied. For $A=134$ these two states become completely occupied in HF. The pairing now scatters some neutrons in the state $3 p 1 / 2$ which is empty in HF. The global effect is a very weak enhancement of the neutron radius in HFB. In conclusion, in $\mathrm{Zr}$ isotopes the pairing correlations can reduce or increase the neutron radii according to the relative occupancy of the weakly bound states close to the Fermi level.

We complete our analysis by considering the $\mathrm{Ni}$ isotopes close to the neutron drip line. The anti-halo effect in $\mathrm{Ni}$ isotopes was discussed already in Ref. [5] by using the Skyrme force SIII. For this force the neutron drip line is extended up to the nucleus ${ }^{88} \mathrm{Ni}$. This prediction is very different from the RHB calculations, which give ${ }^{98} \mathrm{Ni}$ as the last bound nucleus. Interestingly enough, the same position of the drip line, i.e. ${ }^{98} \mathrm{Ni}$, is predicted by the force SkI4.

The neutron radii of $\mathrm{Ni}$ isotopes calculated with the SkI4 force are shown in Fig. 10. For the Ni isotopes analyzed here the most important single-particle states are $3 s 1 / 2,2 d 3 / 2$ and $1 g 7 / 2$. The first two states are weakly bound while $1 g 7 / 2$ is a resonance state in ${ }^{86} \mathrm{Ni}$ and ${ }^{88} \mathrm{Ni}$ and becomes weakly bound beyond ${ }^{88} \mathrm{Ni}$. This level structure is different from the case of $\mathrm{Zr}$ isotopes in

\begin{tabular}{|c|c|c|c|c|c|c|c|}
\hline$A$ & 86 & 88 & 90 & 92 & 94 & 96 & 98 \\
\hline $3 s 1 / 2$ & 2 & 2 & 2 & 2 & 2 & 2 & 2 \\
\hline $2 d 3 / 2$ & 0 & 2 & 4 & 4 & 4 & 4 & 4 \\
\hline $1 g 7 / 2$ & 0 & 0 & 0 & 2 & 4 & 6 & 8 \\
\hline
\end{tabular}

TABLE II: Number of neutrons in the states $3 s 1 / 2,2 d 3 / 2$ and $1 g 7 / 2$ corresponding to $\mathrm{Ni}$ isotopes and $\mathrm{HF}$ calculations with the SkI4 force.

which all the states involved in the anti-halo effect are bound states.

Coming back to the neutron radii shown in Fig. 10, we can see that, from ${ }^{86} \mathrm{Ni}$ to ${ }^{94} \mathrm{Ni}$ the $\mathrm{HF}$ radii are larger than the HFB ones, the difference being maximum for ${ }^{90} \mathrm{Ni}$. As in the case of $\mathrm{Zr}$ isotopes, the effect of the pairing correlations upon neutron radii ca be simply traced back to the occupancy of the HF levels shown in Table II. The most important thing we can notice in Table II is that the state $3 s 1 / 2$ is fully occupied in $\mathrm{HF}$ for all the considered isotopes. Thus in all these nuclei the pairing force will depopulate the state $3 s 1 / 2$, which has the largest spatial extension compared to the other high- $l$ states shown in Table II. This explains why in Ni isotopes the $\mathrm{HF}$ radii are systematically larger than the HFB radii.

\section{CONCLUSIONS}

In this work we have examined the evolution of the nuclear structure of $\mathrm{Zr}$ isotopes at large neutron excess, with a special attention to the far out region of neutron densities. This is motivated by the predictions of the RHB approach which indicate the presence of a giant neutron halo in these nuclei. We have therefore used a different approach, namely the Skyrme-HFB model. We find that, in this isotopic chain the presence or absence of giant halos depends essentially on the location of the predicted neutron drip line. Thus, there is a strong model dependence in this type of study as illustrated by the results obtained with the Skyrme forces SLy4 and SkI4.

For the drip line to be displaced towards heavier isotopes, a necessary condition is that some HF orbitals become bound when A increases. In this case additional bound neutrons can be accommodated and bound nuclei of heavier mass can be formed. An illustration of this situation is provided by the $\mathrm{Zr}$ isotopes where this necessary condition is fulfilled by some model like SkI4 but not by SLy4. Once the necessary condition is realized, a neutron halo may exist if some of the weakly bound HF orbitals correspond to low angular momenta (3p3/2 and $3 \mathrm{p} 1 / 2$ in the case of Zr with SkI4) so that the centrifugal barrier is weak enough to let the wave functions extend far out.

Thus, the decisive factor is the HF mean field while the pairing correlations play a lesser role. Which part 
of the mean field governs the position of the drip line is not clear, and this point deserves further study. On the example of Zr with SkI4 and SLy4 it seems that the main role cannot be attributed to the spin-orbit potential but rather to some part of the mean field which influences the relative positions of $1 \mathrm{~h}$ and $3 \mathrm{p}$ orbitals.

We have also seen that the pairing correlations can lead to the anti-halo effect, as it is well known, but it can also sometimes produce an enhancement of the halo effect. This can be understood by analyzing the occupation probabilities of the least bound $(l j)$ orbitals. Finally, it can be noted that the anti-halo effect can occur even when all active orbitals are bound.
[1] Proceedings of the 17th International Conference on Cyclotrons and their Applications (october 2004), Tokyo.

[2] M. Bender, P.-H. Heenen, and P.-G. Reinhard, Rev. Mod. Phys. 75, 121 (2003).

[3] J. Meng, H. Toki, S.G. Zhou, S.Q. Zhang, W.H. Long, and L.S. Geng, Prog. Part. Nucl. Phys. (2006, in press) (arXiv: nucl-th/0508020).

[4] J. Dobaczewski, H. Flocard, and J. Treiner, Nucl. Phys. A 422, 103 (1984); J. Dobaczewski, W. Nazarewicz, T.R. Werner, J.-F. Berger, C.R. Chinn, and J. Dechargé, Phys. Rev. C 53, 2809 (1996).

[5] M. Grasso, N. Sandulescu, Nguyen Van Giai, and R.J. Liotta, Phys. Rev. C 64, 064321 (2001).

[6] J.Meng and P. Ring, Phys. Rev. Lett. 77, 3963 (1996); J. Meng, Nucl. Phys. A 635, 3 (1998).

[7] J. Meng, Phys. Rev. C 57, 1229 (1998); M. Del Estal, M. Centelles, X. Viñas, and S.K. Patra, Phys. Rev. C 63, 044321 (2001).
[8] J. Terasaki, S.Q. Zhang, S.G. Zhou, and J. Meng, arXiv: nucl-th/0603005.

[9] J. Meng, H. Toki, J.Y. Zeng, S.Q. Zhang, and S.-G. Zhou, Phys. Rev. C 65, 041302 (2002).

[10] J. Meng and P. Ring, Phys. Rev. Lett 80, 460 (1998).

[11] S.Q. Zhang, J. Meng, H. Toki, I. Tanihata, and S.G. Zhou, Science in China G 46, 632 (2003) (arXiv: nucl-th/0302032) .

[12] N. Sandulescu, L.S. Geng, H. Toki, and G.C. Hillhouse, Phys. Rev. C 68, 054323 (2003).

[13] J.-F. Berger and M. Girod, private communication.

[14] P.-G. Reinhard and H. Flocard, Nucl. Phys. A 584, 467 (1995).

[15] S.A. Fayans, S.V. Tolokonnikov, and D. Zawischa, Phys. Lett. B 491, 245 (2000); K. Bennaceur, J. Dobaczewski, and M. Ploszajczak, Phys. Lett. B 496, 154 (2000). 